\title{
Vehicle as a Witness using Video Capturing
}

\author{
Salah Tofiq Alshami \\ Author \\ Department of Computer Science and \\ Engineering, Andalus University for Science \& \\ Technology, Yemen
}

\author{
Fekri M. Abduljalil \\ Supervisor \\ Department of Computer Science, Faculty of \\ Educ., Arts, \& Science, University of Sana'a, \\ Sana'a, Yemen
}

\begin{abstract}
Several technologies are organized to maintain and help Intelligent Transportation System (ITS). Wireless communications are widely used for short and long range communication within ITS. Sophisticated sensors estimate the speed, length, and class of vehicles and the distance between them and more. Video and internet are also share their resources with the ITS. However road accidents and events are increased especially in modern cities. These events such as fires, stealing, explosions and others are also need to be recorded using new applications that concern on shrinking the bad results of these accidents and events.

In this paper, we intend to make Vehicles act as a video Witnesses for all events on roads using vehicular networks and cloud computing.

The proposed scheme solves the problem of how to make a vehicle work as a witness when an event or an accident is occurring with storage saving. It enables user to get video of vehicle accident and road's events anytime anywhere. The scheme is implemented using test bed and its performance is evaluated.
\end{abstract}

\section{Keywords \\ Video Capturing}

\section{INTRODUCTION}

Nowadays Cloud computing 'which is known as services delivery such as shared resources, and platforms in the interest of end-users' is used for the Intelligent Transportation Systems (ITS) to improve transport outcomes such as road safety, travel reliability and informed travel choices [1]. Cloud computing can perfectly work with smart cities that need more enhancement for all its aspects one of them is (ITS) for the reason of reduce the cost of traffic and accidents and to bring safety roads. The purpose of the Smart Cities is to drive economic growth and improve the quality of life of people by enabling local area development and harnessing technology, especially technology that leads to Smart outcomes.

Few years ago, a technology that has received much attention is the Vehicular Ad-Hoc Network (VANET). A VANET is a set of moving vehicles in a wireless network that apply the Information Communication Technology (ICT) [2].

To bring the best for people you should look for the best solutions. As we all know people needs to avoid accidents. They also wants to know about events on the roads. The Intelligent Transportation system must offer efficient vehicular accident management [3] because the events and accidents in transportation has daily increased for example around the world 1.2 million traffic death, 50 million injuries, In China, 500,000 accidents [1].

Smart vehicle become very sophisticated with many sensors that can get vehicle speed, time/date, direction, \& detailed driving behaviors such as harsh braking, fast acceleration, hard turning, location, and it has connection to the internet and it could connect with other smart vehicles.

Smart vehicles can work as a witness for events and accidents with the help of ITS, Cloud Computing, VANET, and vehicular network to introduces a new service mechanism.

In this paper, we propose a service that use mounted cameras on the vehicles to provide video witness for all events in the roads. The vehicular nodes serve as witnesses to the event and provide the forensic evidences to the law enforcement agencies for investigation [4].

The proposed scheme apply video recording for all events on the roads. It gives a solution to the problem of limited storage space.

The remainder of this paper is organized as follows. In Section 2, related works are presented. The proposed schemes are introduced in Section 3. Section 4 presents security. Section 5 presents the system implementation. Conclusions in Section 6. Finally the references in section 7 .

\section{RELATED WORK}

A Vehicular Cloud service that delivers images on demand to citizens by using vehicles' on board cameras.

Gerla et al [5] proposed a cloud-based service (Pics on Wheels) that delivers lively images for a specific area on demand by query from a customer using the vehicle's cameras. In their scheme, anybody can query the cloud and take photo for anywhere inside the scope of the service so it is a location based service.

The Pics-on-Wheels service selects a group of vehicles to take photo shots of a given urban landscape within a given timeframe as requested by a customer.

This service need the vehicles to register in the centralized cloud manager and upload their own GPS location periodically to the cloud manager.

These images can then be used for knowing what has happened at the time of the accident, also this service can help for forensic and assurance claims purpose.

The previous scheme uses images. The results of images are very weak compares with the results of video. It is impossible for the image to cover the whole scene of the events. This scheme needs perfect conditions to provide the service. Conditions like the speed of vehicles, the direction of vehicles and others. Add to that, small mistakes make the service useless such as wrong location query or weak signal of internet.

In research paper [6], a real-time video capture of vehicle 
accident is proposed. The proposed scheme solves the problem of huge storage needed for recording vehicle accident in the smart vehicle and in the remote ITS server. It just records the last period before and during the accidents. The vehicle records the video from inside the vehicle that crashed.

The author introduce two methods for accident detection. The first one is when the vehicle turnover (VTD). The second detection method is called vehicle crash detection (VCD). The scheme of recording video in this paper is to use two video files. It starts recording in one file for $\mathrm{T}$ minutes, then starts record in the second file for T minutes. After the T minutes of recording in the second file is finished, it removes the contents of the first file and starts recording to it. Then the above steps are repeated until accident occurs.

There are two problems in this scheme first the deleted video cannot be recovered, so if you want it you will not be able to get it, because it removed permanently. The second problem is the scheme proposed the vehicle recorded video from its own camera and this cannot give a clear and exact video for the accidents and maybe the camera will be crashed because the accident.

In [7], it proposed a new VANET-cloud service called VWaaS (Vehicle Witnesses as a Service) in which vehicles moving on the road serve as anonymous witnesses of designated events such as a terrorist attack or a deadly accident. When confronted the events, a group of vehicles with mounted cameras collaborate with roadside stationary cameras to take pictures of the site of interest (SoI) around them, and send the pictures to the cloud infrastructure anonymously. The pictures are sent to the cloud in a way that the privacy of the senders can be protected, and kept by the cloud for future investigation.

Once a designated event occurs, the vehicular nodes serve as witnesses to the event and provide the forensic evidences to the law enforcement agencies for investigation.

In [7], there is no way of detecting the accident which the author said that "Note that we assume the existence of a mechanism for the VANET to detect the occurrence of a designated event since it is out of the scope of this paper".

Also the previous scheme uses images. The pictures is not enough witness it cannot give details. Compare with video it can provide more details.

Unfortunately the author gives a topic and he concentrates on other things. The main idea in the paper is the security and he doesn't talk about vehicle as a witness but within narrow limits.

In this paper, video cover the whole event because it continually recording everything.

Many papers talk about automatic detecting accidents in different ways and methods. For example [8] uses seven cameras at two locations for detection. Accident detecting device: Receives images from cameras, processes them and detects accidents and Stores moving images in memory, and send them to traffic center.

In [9], authors said that accidents can be detected precisely with the help of both Micro electro mechanical system (MEMS) sensor and vibration sensor. Whenever an accident occur the system detect it and send message through GSM module to rescue team and police station. They supposed that the message will be sent within a few seconds covering geographical coordinates, the time and angle of the vehicle.
In [10], the paper give a new way for detecting an accident which use smartphone with some sensors to detect the accident and take a pictures for it and determine the location, after that it upload these information to the server. The server will send them to the aid team to give immediately assistance.

\section{PROPOSED VIDEO WITNESS AS A SERVICE IN VCC}

In this section, the proposed witness system is presented. The proposed system are presented in three parts. First, describe system model. Then, introduce system functions. Finally, provide some scenarios for system model.

\subsection{System Model}

Figure 1 shows the architecture of the proposed system. It consist of 4 main components:

1. Service seeker.

2. Vehicle
a. Location detector
b. GPS receiver.
c. Modem for
d. WIFI 802.11n.
e. Or 3G connection.
f. Camera.
g. Sensors.
h. Application client.

3. Server

a. Application service provider (ASP).

b. Database.

4. Road side units.

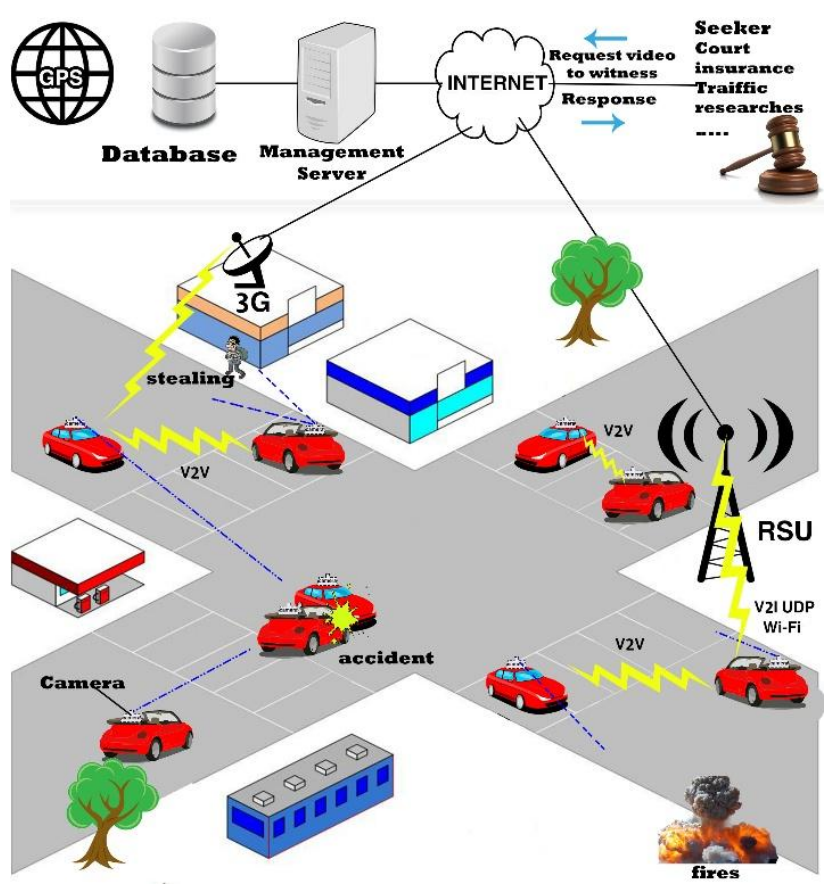

Fig 1 Proposed System Model

\subsubsection{Service seeker}


It is a customer or a client who query for a video for specific location on the road through request message. The request message consist of location, date, time, direction of lane. Add to that, service seeker could be trial, insurance, traffic police, researches, police investigator, and others. These section needs a witness for events or accidents.

\subsubsection{Service seeker}

It has five sub components according to our system:

1. Location detector: it is a means for automatically determining and transmitting the geographic location of a vehicle. There are many application and systems that detect location. Most commonly is Global Positioning System or GPS. It is also low price and ubiquity. It just needs only a receiver to collect signals from the satellite segment. We can use GPS for every vehicle to collect location data periodically (every 100 meres) then upload location data to the server. RoadTrack [11] suggest that vehicle updates shouldn't be faster than minutes per vehicle, or the spectrum becomes congested.

2. Modem it is a device that connect to the internet whether through Wi-Fi $802 . n 11$ or $3 \mathrm{G}$ or anything else that connect to the internet. It is also connect to other vehicles through VANET.

3. Camera we suggest to use omnidirectional camera. It is a camera with a 360-degree that covers (approximately) the entire sphere. We suggest to be in the top of the vehicle. It works while the vehicle is moving.

4. Sensors it is small devices on vehicle that can get vehicle speed, time/date, direction, \& detailed driving behaviors such as harsh braking, fast acceleration, hard turning.

5. Application Client: it is in-vehicle computer which controls:

a. Sending and receiving messages like registration message, location update message, video, v2 v messages etc.

b. Processing video it has many functions like store, update, delete, add, cut, start, stop, and others.

c. Get location it uses GPS device to get the location of vehicle and store it.

d. It communicate with other vehicles through VANET once accident is occur.

\subsubsection{Server}

1. Application service provider (ASP): it is the target server that manage to act as a broker between the vehicle and the Service Customer. It manages participating vehicles in the mobile cloud and keeps track of location updates for each vehicle and schedule them in the database. It is also receive video from vehicles and store it in the database. It uses web services to communicate with the edge points of the system.

2. Location \& video database: It is an organized collection of data that store all information about vehicles in the system. Vehicle ID and its location, time, date, direction and the requested video are the most important data of the system. The database has a collection of schemas, tables, queries, reports, views, and other objects that help with storing and retrieving data using DBMS.

A database management system (DBMS) is a computer software application that interacts with the user, other applications, and the database itself to capture and analyze data [12].

\subsubsection{Road Side Units (RSU)}

It is devices located on the roadside. They provides connectivity support to passing vehicles. Figure 2 shows the components and the interaction between each other.

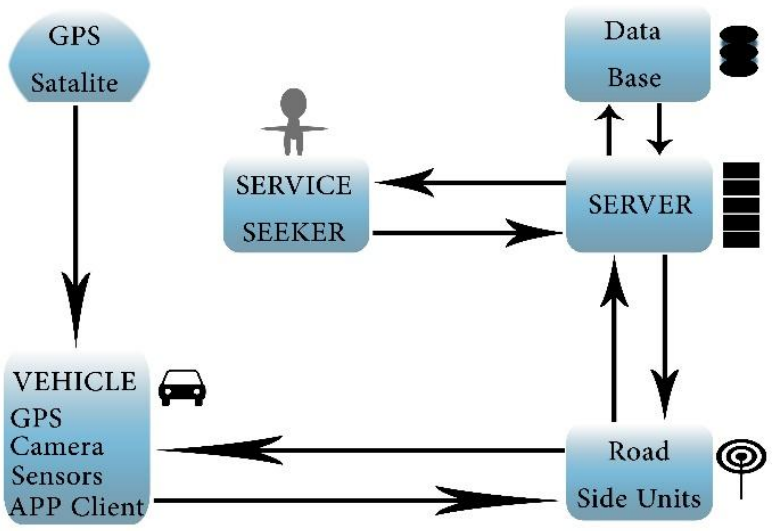

Fig 2 the Interaction of Components

\subsection{The proposed system design}

This section explains how the system works.

\subsubsection{Registration}

First of all, vehicle must register in the server of the system by sending its ID and other information to the server. The server by his turn will create records for this vehicle in the database of the system. The communication will be done through web services. Web services may use SOAP (Simple Object Access Protocol) over HTTP protocol. The identity of the vehicle will be secret.

\subsubsection{Location Update}

First application client get the GPS coordination of the vehicle using GPS Tracking Device.

After that, application client save the GPS coordination in the hard drive of the vehicle. It repeat the process of getting GPS coordination of the vehicle every one minutes but if the vehicle's speed less than $6 \mathrm{Km}$ per hour it could get GPS coordination of the vehicle every 100 meters.

In [13] the author describe the communication way between mobile station and network with details. He mentions the update location management, and handoff process. Update location process may be periodically or, on location area boundary crossings.

Location update could be Time-based Update, Movementbased Update, or Distance-based Update.

Location update message (LUM) is a message that contains many parameters such as vehicle Id, location (X, Y, and Z), 
date, time and direction. (LUM) is the message that will be uploaded to the server periodically.

\subsubsection{Video recoding}

The omnidirectional camera is working while the vehicle is on. It records stream of video and it covers everything in the road. The video is saved in the storage of the vehicle. It use full time recording "if the storage of the video become full it delete from the end to allow more space to add new video to the start" figure 3 .

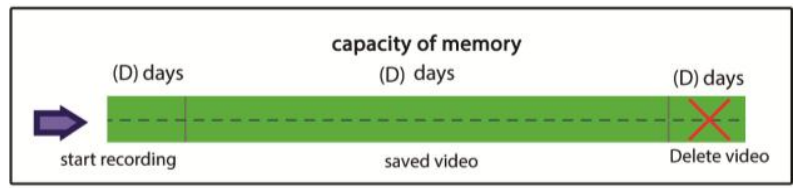

Fig 3 Full time recording (First in First out)

Figure 3 full time recording

Here is the algorithm of video recording and another one for accident detecting.

Algorithm 1: video recording

Input: file, Time, Date

Begin

While (engine is on)

Continue record video to file in the storage

Add information about time and date to the video files

If the secondary storage is almost full then

Delete (T) Time from the end of the video

Continue recording

End

If an accident occur to any vehicle, it send a sign to around vehicles. As soon as the around vehicle receive the sign they will cut $\mathrm{T}$ minutes from their saved video before the time of the received sign and $\mathrm{T}$ time after it. Then they upload this small video to the storage of the server with the vehicle's information.

\section{Algorithm 2: if accident}

Input: file, Time, Date

Begin

While (engine is on)

If there is sign of an accident received then

Cut small file with $\mathrm{T}$ minutes before and $\mathrm{T}$ minutes after received sign

Upload the file to the server with vehicle information

End

\subsubsection{Accident Detecting}

There are three steps for accident detection:
First, there are many types of accidents such as roll over or hitting on wall or on other vehicles. When an accident occur to vehicle1 its accident sensors starts on. The application client receives accident signal from accident sensor.

Note that the vehicle1 and the around vehicles are recording video.

Second, as soon as application client of vehicle1 receive accident signal it cut accident video from its storage. Accident video is $\mathrm{T}$ time before and $\mathrm{T}$ time after the receiving accident signal. Also the application client broadcast message to around vehicle with details of accident. The around vehicle cut accident video from their storage.

Finally the vehicle 1 and around vehicles upload the accident video to the server with some information like location and time of accident. The server will store accident videos in the database for the need of witness or client. 

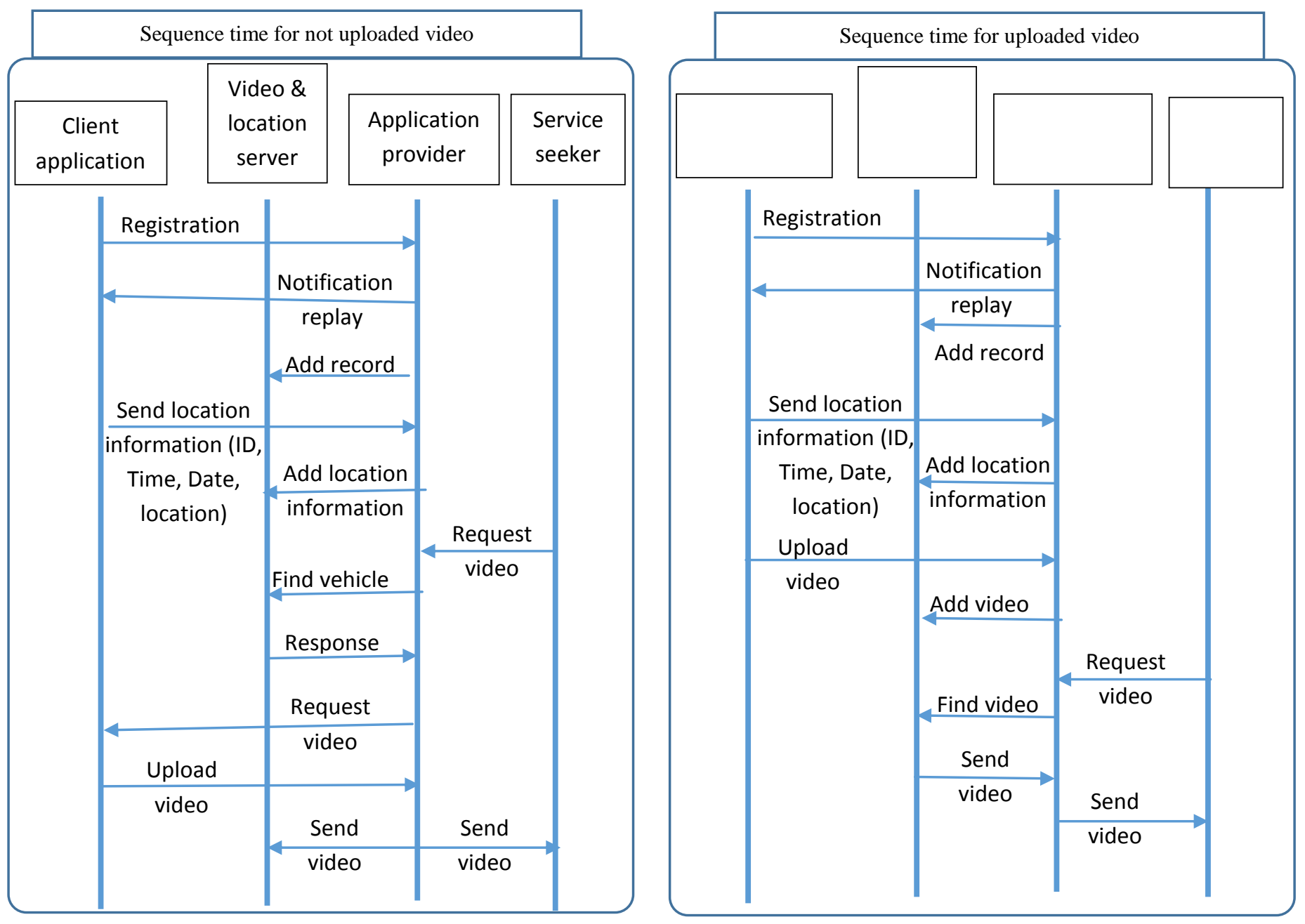

Fig 4 Sequence Time for Uploaded and Not Uploaded Video

\subsubsection{Locate Video Witness}

Video witness is the video which the client need it. The process starts from the client which he query for a video at specific time and location.

The server searches for the requested video witness in the database. If the server found a match it returns the results to the client. But if the server doesn't find a match it searches for the vehicles that pass the requested location in the database. When the server get some vehicles it send a message to these vehicles. The message ask the vehicle to get a video witness from vehicle database according to given location and time.

As soon as the vehicles get the message they cut the requested video and upload it to the server. The server by his turn returns the results to the client. If it is important witness and we couldn't to get video witness we can take the information of the vehicles then we goes to them and take the video manually.

\section{SECURITY}

The goals of this section are:

- $\quad$ Hide the identity of the witness.

- Protect the vehicle database and server database from hackers.

- $\quad$ Save the data from man in the middle attacks.

First hiding the identity of the witness 'which is the vehicle with its owner' is very important goal. Because if it is possible to know who is the witness easily he will be probably bothered by who witness against them. So our duty is to hide the identity with the possibility of retrieve it if we need. To do that we suggest to use way of Rasheed Hussain et al which they used pseudonym for each vehicle. The pseudonym will be exchanged automatically every period of time. Also they will be encrypted. Add to that we can revoke them in the need as it described in [7].

Second protect data in the server and in the vehicle using powerful passwords, firewalls and antivirus. Beside that we want to prevent any hackers who want to change or steal data using encryption with strong algorithm such as RSA and hash function.

Use a strong encryption between the client and the server. In this case server authenticates client's request by presenting a digital certificate, and then only connection could be established.

Security is from the most important parts of any system and our system is not exception so we suggest a security plan consist of three parts the first one is to protect the storage device in the vehicle by putting the hard drive in strong and unbreakable container. Then we have to encrypt the video stream with a public key which we can use a private key to decrypt the video and because the video is a heavy data we need a method that decrease the processes on video encryption so we can use a partial encryption in [14] the 
authors introduce a way for secure transfer video using an encryption method. They called it partial encryption algorithm (PEA) which provide high security, computational efficiency and compression efficiency. They suggest:

1. Record video.

2. Cut video to frames.

3. Classify frames to odd and even.

4. Encrypted the even frames with AES encryption algorithm.

5. Use a shuffle algorithm to shuffle the odd and even frames using shuffling key.

6. Finally send the video.

Finally the encrypt video will be uploaded to the server and when the service seeker need a video he can contact the server and it will decrypt the video with the private key and send it to the service seeker.

\section{IMPLEMENTATION}

This section introduce simple prototype of the system model. This section has two parts, system setup and results discussion.

\subsection{System Setup}

The components of our prototype are:

Part one Hardware

1. The server (laptop 1): it is installed on HP laptop with Intel core i5 and 4GB of ram with windows 7.

2. The vehicle (laptop 2): it is installed on Lenovo laptop with Intel Core (TM) i5 $2.40 \mathrm{GHz}$ and ram of 6 GB with windows 7 Pro 64 bit and 500GB hard drive.

3. Normal vehicle.

4. Smart phone galaxy S3 4.4.2 with 16GB internal memory and ram 2GB and 8 mega pixels photo and 1920x1080 mega pixels video camera and GPS. It has three tasks:
a. It records video and save it in its internal memory.
b. It gets and registers GPS points every 100 meters and also it takes time, and date. Add to that it calculated and registered speed and distance.
c. It sends the video and the other information to the laptop2 (vehicle).

We suggest to use Wi-Fi Broadcom 802.11n access point on the road. The net data rate is $54 \mathrm{Mbit} / \mathrm{s}$ to $600 \mathrm{Mbit} / \mathrm{s}$. it uses multiple antennas to increase data rates. $802.11 \mathrm{n}$ offers 70 meters indoor range and 250 meters outdoor. If we setup our system in a road with five kilometers we will need 20 antennas of $802.11 \mathrm{n} \mathrm{Wi-Fi} \mathrm{Broadcom.} \mathrm{If} \mathrm{we} \mathrm{upload} 100 \mathrm{Mb}$ data to the internet using $802.11 \mathrm{n} \mathrm{Wi-Fi} \mathrm{Broadcom} \mathrm{we} \mathrm{will}$ need less than two seconds. We can make our system to be auto connected to the antennas.

Part two Software
1. TCP/IP and UDP protocols.
2. FFMPEG program for media [15].
3. .net frame work 4.5 with $\mathrm{C} \#$ programming

language.

4. "GPSTracker lite" application from google paly store.

5. Windows 7.

6. Management program for server and vehicle figure 6 . We wrote this program using c\#. It has two sides one for server and the other for vehicle.

The vehicle (laptop 2) is connected with server (laptop 1) using wireless network Broadcom 802.11n. The vehicle (laptop 2) sends the registration information to the server (laptop1). It takes the information from the smart phone and save them in its hard drive. The vehicle (laptop 2) has a program with $\mathrm{C \#}$ language which has two methods of dealing with the video and the other information as follows.

Method 1, we simulate an accident by using button with click event. As soon as we click on the button the program cut one minute from the video before the time of clicking and one minute after the time of clicking. We used ffmpeg [15] program to deal with media like cutting video. After that it sends the video and the other information to the server (laptop1).

Method 2, vehicle (laptop 2) receives a request from the server (laptop 1) which asks a video with specific location, date and time. The vehicle (laptop 2) receives the request and it use ffmpeg program to cut the need video. Vehicle (laptop2) sends the need video back to the server (laptop 1).

The server (laptop 1) is supposed to manage all the processes like registering, connecting with the clients, saving the video and the other information. It also update the location information and so on.

We used socket programming with (IP, TCP, and UDP protocols) for the communication between vehicle (laptop 2) and server (laptop 1).

How to get video in specific time?

To get video in specific time and specific location we suggest the following steps:

1. The seeker of the video will ask apart of video for specific location and time.

2. The server tell the seeker to enter location and time.

3. The server takes the information and creates a grid of locations points with offset of 100 meters around the token point.

4. The server search in this grid with the token time.

5. If it get vehicles it will contact with them to have the video.

6. The vehicle replied with the needed video.

7. The server send it back to the seeker.

\subsection{System Scenario}

The vehicle moved from Darsalm circle (15.277305, 44.234292) to Baitbous circle $(15.277587,44.220014)$ the distance was about $1.6 \mathrm{~km}$. as shown in map 1 . It was on Saturday 24-12-2016 from 13:22 to 13:26 at noon. The smart phone record video with length of 00:04:33 and size of 557 MB. The video has been saved in the memory of the smart phone.

We translated the recorded video to the labtop2 (vehicle). 


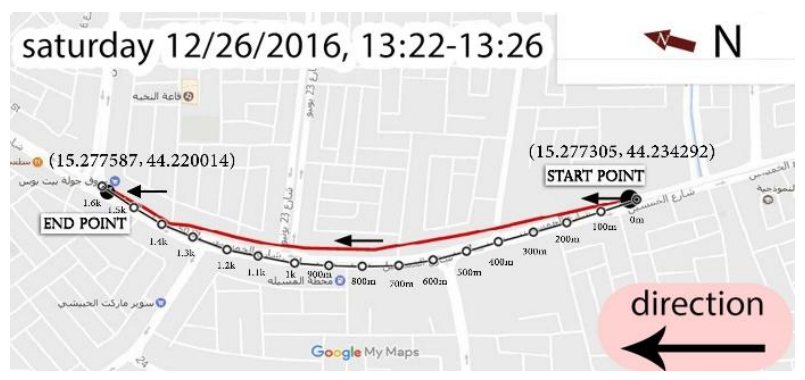

Fig 5 Map of the Vehicle Moves between Two Points

We set up a wireless ad hoc computer to computer network between laptop1 (server) and laptop2 (vehicle). And by using management program we made two scenarios:

First the vehicle contact with the server. The server ask video with 2 minutes length. The vehicle cut the 2 minutes video and send it back to the server.

Second we simulate happening of accident. As soon as the simulation starts the vehicle cut video with 2 minutes length and upload it to the server.

\subsection{Results Discussion}

This section will describe an approximately results of our simple system that we built.

\subsubsection{Storage analysis}

\section{First the storage in the vehicle}

The vehicle has hard drive with size of minimum 2 terabyte. We suggest to use cameras with H.264 encoding with $20 \mathrm{fps}$ and $1280 \times 1024$ resolution. That will give us 40 days of video recording in two terabyte hard drive [16]. If we know that the vehicle will work at maximum 12 hours per day, that mean the days of recording video will duplicate to 80 days per day 12 hours. The storage of all videos will be distributed between all vehicles in the system.

\section{Second the storage in the server}

Server will not use very big storage. The reason behind that is in two points:

First server will get information from vehicles periodically. This information include the Id of vehicle, its location, time, and other information. If we supposed that the size of these information is 100 byte.

Also suppose vehicle goes around with distance of $50 \mathrm{KM}$ per day as average. If we know that the vehicle update its information every 100 meter. That mean it will do update 500 times per day. As we mentioned the size of updated information is 100 bytes (we exclude the size of video data for now) that mean the vehicle will upload the size of 50000 byte (48.828 KB) per day.

Suppose that there are 100000 vehicle goes around per day in a normal city that mean the size of uploaded data will be 5000000000 byte $(4.6566 \mathrm{~GB})$ per day.

Finally if we know that Dropbox stores a total of 6.7GB per second [17], we admit that this system use very small space of memory.

The second point is the storage of video data. Since the video data is very big we need a method to decrease the amount of video that will be uploaded to the server.

The method is to upload the need video. The need video is the recording of important events on the road such as accidents or the video that asked by customer for specific area which is from one to three minutes.

\subsubsection{Final results:}

As shown in system scenario the vehicle has already stored video inside its storage.

We test the time needed for performing a service.

First, a job request is initiated from the Sever, which request for video at specific location, date and time. Once the vehicle receives the request, it immediately returns an acknowledgement to the Cloud Service Provider. Then, it takes the information of the video like location time and date and cut the need video, after that it upload the video to the Cloud Service Provider

Figure 4 shows the time needed for upload short video in the experiment. The time between a job request and data received varies between three to five seconds. However, the major delay happens on the video uploading process, which generally takes up to three seconds to complete. And cutting time less than one second. The transmission delay to upload $150 \mathrm{MB}$ video is about four to five seconds

It cut video of 2 minutes and $150 \mathrm{MB}$. We send it from the vehicle (labtop2) to the server (labtop1) it takes less than 4 seconds.

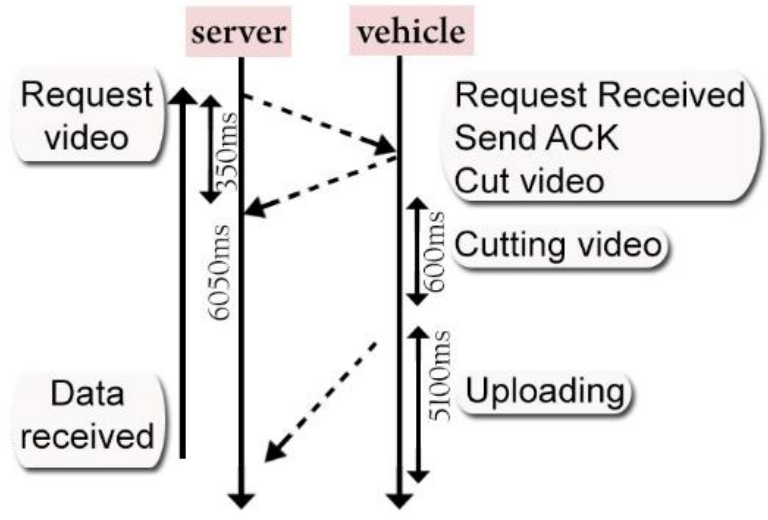

Fig 6: Time Need for Upload Short Video

Relationship between size of video and transfer time

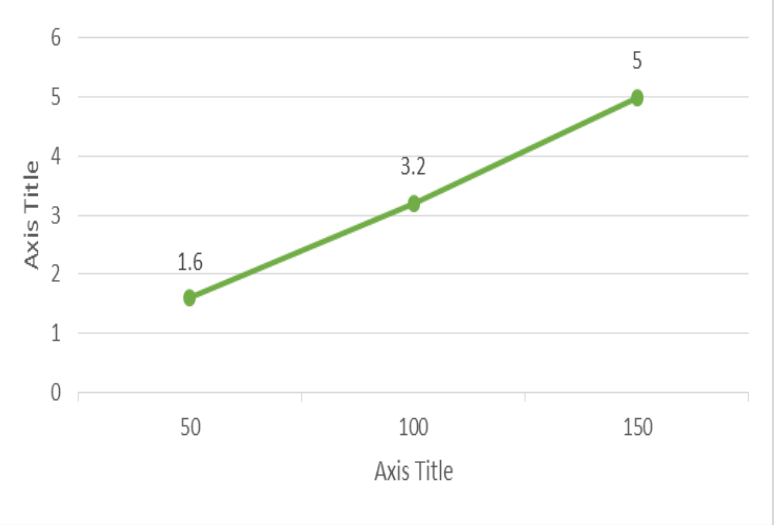

Fig 7: Relationship between Size of Vide and Transfer Time 


\section{CONCLUSION}

With many techniques our life is improving gradually as we seen we introduce a technique that act as a witness in roads. In this paper we made the on roads vehicles emulate humans' actions with capture a video for events on roads and save them in a server for the future need. This help Intelligent Transportation Systems (ITS) to improve transport outcomes such as road safety, travel reliability and informed travel choices. With many sources that make this work possible like VANET, smart vehicle, cloud computing and others we give a great service for the world.

Accordingly, next stage for the research will be pursued in indoor for improving the architecture using simulator and simulations of the network architecture will be implemented under riverbed Network Simulators. Furthermore, we will improve the research to give more services

\section{ACKNOWLEDGEMENTS}

A substantial part of this research was financed by Alandalus University, Republic of Yemen. I am grateful to University of Alandalus for having believed that this might be a real and reasonable investment.

\section{REFERENCES}

[1] Alifia Fithritama, Ramya Narayanan "Intelligent Transportation System (ITS) using Cloud Computing" Course: Cluster, Grid, and Cloud Computing, Lecturer: Andrey Y Shevel, Date: 5 June 2014.

[2] Md. Whaiduzzaman a,n, MehdiSookhak a, AbdullahGani a, RajkumarBuy"A surveyonvehicularcloudcomputing" Mobile Cloud Computing Research Lab, Faculty of Computer Science \& Information Technology, University of Malaya, 50603 KualaLumpur, Malaysia

[3] Fekri M. Abduljalil "A NOVEL REAL-TIME VIDEO AND DATA CAPTURE OF VEHICULAR ACCIDENT IN INTELLIGENT TRANSPORTATION SYSTEMS" International Journal of Computer Networks \& Communications (IJCNC) Vol.6, No.2, March 2014.

[4] Rasheed Hussain, Fizza Abbas, Junggab Son, Donghyun Kimy, Sangjin Kimz, and Heekuck Oh." Vehicle Witnesses as a Service: Leveraging Vehicles as Witnesses on the Road in VANET Clouds" DOI: 10.1109/CloudCom.2013.64 Conference: IEEE CloudCom, At Bristol, UK.

[5] M. Gerla, J.-T. Weng, and G. Pau, "Pics-on-wheels: Photo surveillance in the vehicular cloud," in Computing, Networking and Communications (ICNC), 2013 International Conference on. IEEE, 2013, pp. 11231127.

[6] Fekri M. Abduljalil "A NOVEL REAL-TIME VIDEO AND DATA CAPTURE OF VEHICULAR ACCIDENT
IN INTELLIGENT TRANSPORTATION SYSTEMS" International Journal of Computer Networks \& Communications (IJCNC) Vol.6, No.2, March 2014.

[7] Rasheed Hussain, Fizza Abbas, Junggab Son, Donghyun Kimy, Sangjin Kimz, and Heekuck Oh." Vehicle Witnesses as a Service: Leveraging Vehicles as Witnesses on the Road in VANET Clouds" DOI: 10.1109/CloudCom.2013.64 Conference: IEEE CloudCom, At Bristol, UK.

[8] A.ISuge, H.Takigawa, H.Osuga, H.Soma, and K.Morisaki "ACCIDENT VEHICLE AUTOMATIC DETECTION SYSTEM BY IMAGE PROCESSING TECHNOLOGY" 1994 Vehicle Navigation \& information Systems Conference Proceeding.

[9] C.Prabha , R.Sunitha , R.Anitha "Automatic Vehicle Accident Detection and Messaging System Using GSM and GPS Modem" International Journal of Advanced Research in Electrical, Electronics and Instrumentation Engineering (An ISO 3297: 2007 Certified Organization) 7, July 2014

[10] Chris Thompson, Jules White, Brian Dougherty, Adam Albright, and Douglas C.Schmidt " Using Smartphones to Detect Car Accidents and Provide Situational Awareness to Emergency Responders " Vanderbilt University, Nashville, TN USA

[11] P Pesti, L Liu, B Bamba, A Iyengar, M Weber "ROADTRACK: Scaling Location Updates for Mobile Clients on Road Networks with Query Awareness", Proeeding of the VLDB Endowment, Volume 3, Issue 12, September 2010.

[12] https://en.wikipedia.org/wiki/Database available in $5 / 3 / 2017$.

[13] James Cowling "Dynamic Location Management in Heterogeneous Cellular Networks" A thesis submitted in partial fulfillment of the requirements for the degree of Bachelor of Computer Science and Technology (University of Sydney Australia) October 2004.

[14] Neethu. J, et al " Secure Video Transfer" CSE at Sreepathy Institute of Management and Technology \& Calicut University, Kerala, India, February 2015

[15] https://ffmpeg.org/.

[16] Seagate " Video Surveillance Storage:How Much Is Enough" www.seagate.com 2012 Seagate Technology LLC.

[17] https://www.extremetech.com/computing/129183-howbig-is-the-cloud available in $4 / 21 / 2017$. 